\title{
The usage of a lactobacilli probiotic in the non-surgical therapy of peri-implantitis: A randomized pilot study
}

\author{
Isabelle Laleman (i) | Martine Pauwels | Marc Quirynen | Wim Teughels
}

Department of Oral Health Sciences, KU Leuven \& Dentistry, University Hospitals Leuven, Leuven, Belgium

\section{Correspondence}

Isabelle Laleman, Catholic University Leuven, Department of Periodontology, Kapucijnenvoer 33, 3000 Leuven, Belgium.

Email: isabelle.laleman@kuleuven.be

Funding information

This study was partially financially supported by BioGaia AB Sweden. Additional support came from grants from the KU Leuven (C24/17/086) and the FWO (G091218N).

\begin{abstract}
Objectives: Examine the clinical and microbiological benefits of a dual-strain Lactobacillus reuteri probiotic on the non-surgical therapy of initial peri-implantitis. Materials and methods: This randomized, double-blind study targeted patients with initial peri-implantitis, that is peri-implantitis with a maximum mean probing pocket depth of $6 \mathrm{~mm}$ and maximum $3 \mathrm{~mm}$ bone loss compared with loading. A full-mouth prophylaxis was performed and the peri-implantitis sites were debrided. Subsequently, local application of the study drops was carried out at the peri-implantitis sites and the study lozenges were handed out. The patients in the probiotic group received drops and lozenges containing L. reuteri (ATCC PTA 5289 \& DSM 17938), those in the control group received placebo products. At the implant level the measurements of interest were bleeding, probing pocket depth and plaque. Fullmouth bleeding and plaque scores were also recorded. Microbiological samples were taken from the tongue, saliva and subgingivally around the implants.

Results: All clinical parameters were significantly decreased after 12 and 24 weeks. At the implant level the only statistically significant difference was a greater decrease in plaque levels in the probiotic versus the control group ( $p=.002$ at 24 weeks). At the full-mouth level, the only intergroup difference was the greater decrease in fullmouth bleeding on probing sites in the probiotic group compared with the control group ( $p<.001$ at 24 weeks). Concerning the microbiological outcomes, no significant differences could be found at any time point, neither intra- nor intergroup.

Conclusions: No adjunctive effects of the use of $L$. reuteri probiotics in the treatment of peri-implantitis were found.
\end{abstract}

\section{KEYWORDS}

debridement, dental plaque, gingival bleeding on probing, Lactobacilli reuteri, peri-implantitis, probiotics, therapy

\section{1 | INTRODUCTION}

Probiotics are defined as live microorganisms that, when administered in adequate amounts, confer a health benefit on the host (Hill et al., 2014). Their application is very diverse, ranging from gut to oral to even mental health. Currently, dozens of studies examining the effect of probiotics on gum health and disease are available. These showed for example that probiotics can enhance the results of scaling and root planing in periodontitis patients (Ince et al., 2015; Morales et al., 2016; Sajedinejad et al., 2018; Tekce et al., 2015; Teughels et al., 2013; Vivekananda, Vandana, \& Bhat, 2010). This effect was not only seen clinically, that is as improved pocket probing depth reduction, but also microbiologically (Tekce et al., 2015; Teughels et al., 2013; Vivekananda et al., 2010) and at the level of pro-inflammatory 
biomarkers (Ince et al., 2015). Additionally, probiotics decrease gingival inflammation and/or plaque accumulation (Della Riccia et al., 2007; Harini \& Anegundi, 2010; Krasse et al., 2005; Schlagenhauf et al., 2016; Vicario, Santos, Violant, Nart, \& Giner, 2013). However, other studies failed to reproduce these results (Hallstrom et al., 2013; Iniesta et al., 2012; Shimauchi et al., 2008).

Plaque-induced periodontal diseases are not limited to the teeth, but can also occur around dental implants. Peri-implant mucositis is defined as an inflammatory lesion of the soft tissues surrounding an endosseous implant in the absence of loss of supporting bone or continuing marginal bone loss (Berglundh et al., 2018; Heitz-Mayfield \& Salvi, 2018). Peri-implantitis is specified as a plaque-associated pathological condition in tissues around dental implants, characterized by inflammation in the peri-implant mucosa and subsequent progressive loss of supporting bone (Berglundh et al., 2018; Schwarz, Derks, Monje, \& Wang, 2018). Nowadays, this is a hot topic due the high prevalence of peri-implant diseases and the ongoing search for improved therapies, such as probiotics (Galofre, Palao, Vicario, Nart, \& Violant, 2018; Mongardini, Pilloni, Farina, Di Tanna, \& Zeza, 2017). However currently, the studies examining the benefits of probiotics in this indication are scarce (Flichy-Fernandez et al., 2015; Galofre et al., 2018; Hallstrom, Lindgren, Widen, Renvert, \& Twetman, 2016; Mongardini et al., 2017; Peña et al., 2019; Tada et al., 2018).

At this moment, only two (Flichy-Fernandez et al., 2015; Galofre et al., 2018) out of five studies showed an additional positive effect of probiotic usage on peri-implant mucositis (FlichyFernandez et al., 2015; Galofre et al., 2018; Hallstrom et al., 2016; Mongardini et al., 2017; Peña et al., 2019). In contrast, both studies examining probiotic usage in non-surgical peri-implantitis treatment showed more reduction in pocket probing depth and bleeding on probing for the probiotic than test group (Galofre et al., 2018; Tada et al., 2018).

The purpose of this study was therefore to examine the added clinical and microbiological benefits of a dual-strain Lactobacillus reuteri probiotic on the non-surgical therapy of initial peri-implantitis.

\section{2 | MATERIALS AND METHODS}

The Ethics Committee Research UZ/KU Leuven approved the study protocol of this study (s57668), which was conducted according to the principles of the Declaration of Helsinki and was registered prior to the study start at clinicaltrials.gov (NCT02520401). The CONSORT guidelines regarding reporting in randomized clinical trials were followed.

\section{1 | Study protocol}

For this single centre, double-blind, randomized, placebo-controlled study, patients visiting the Department of Oral Health Sciences (University Hospitals Leuven, Belgium) who were diagnosed with initial peri-implantitis were asked to participate. Peri-implantitis was defined as inflammation of the peri-implant mucosa, measured as probing pocket depth (PPD) $\geq 4 \mathrm{~mm}$ with bleeding, accompanied by radiological bone loss (at least $1 \mathrm{~mm}$ compared with the moment of loading). In this study, initial peri-implantitis was defined as an implant diagnosed with peri-implantitis with a maximum mean PPD of $6 \mathrm{~mm}$ (at the implant level) and no more than $3 \mathrm{~mm}$ bone loss measured on intra-oral radiographs (compared with loading). Reasons for exclusion were as follows: uncontrolled periodontal disease, smoking, systemic disorders possibly influencing the treatment results (e.g., diabetes), antibiotic usage the previous 3 months, previous peri-implantitis treatment for the implant included in the study and pregnancy or breastfeeding. Implants with less than $2 \mathrm{~mm}$ keratinized mucosa or with restorative problems were excluded. The results of subjects included in the study, but violating the eligibility criteria during the study (for example due to an antibiotic treatment) were excluded from the analysis. If more than one implant per patient met the study conditions, the implant that was included was determined by drawing lots. An intent to treat analysis was carried out following the "last observation carried forward" principle, including all the patients that at least attended the 12-week appointment without violating the inclusion criteria.

After signing the informed consent, the patients were assigned to the probiotic or control group. This was done based on a randomization list that was made in advance of the study by a computer program (www.randomization.com) according to a 1:1 allocation ratio. Before the start of the study a staff member not involved in the study blinded the study products. These were all packed in identical bottles and containers to ensure the blinding of the examiner and the participants.

\subsection{Outcome measures of interest}

\subsection{1 | Clinical outcomes at the implant level}

The primary outcome of interest was bleeding on probing (BoP) $30 \mathrm{~s}$ after probing with a Merritt-B probe by an experienced periodontist (IL). This was measured at six sites per implant in two ways. Firstly, this was measured as present or not present. Before the start of the study the intra-examiner variability for this parameter was checked: repeated measurements (on 10 patients) an hour apart showed $92 \%$ agreement.

Secondly, the modified sulcus bleeding index (mSBI) described by Mombelli and co-workers (Mombelli, van Oosten, Schurch, \& Land, 1987) was recorded. This index scores the bleeding on a zero to three scale, where 0: no bleeding, 1: isolated bleeding spots, 2: blood forms a confluent red line and 3: heavy or profuse bleeding. Additionally, at six sites per implant the PPD and presence/absence of plaque was noted (dichotomously) (PI).

The 24-week outcomes were used to calculate whether the "desired clinical endpoint", the resolution of the peri-implant inflammation, was achieved. A healthy peri-implant condition was defined as an implant without bleeding on probing (Berglundh et al., 2018). 


\subsubsection{Clinical outcomes at the full-mouth level}

At the full-mouth level the presence of bleeding on probing (fullmouth bleeding score, FMBS) and plaque (full-mouth plaque score, FMPS) was noted at six sites per tooth/implant for all elements present, this was calculated as a percentage of the total sites measured.

\subsection{3 | Microbiological outcomes}

The deepest pocket from the study implant was selected for microbiological subgingival sampling. Prior to this, the supragingival plaque was removed. The subgingival sample was taken with 8 paper points/pockets, which were subsequently placed in $1 \mathrm{ml}$ of reduced transport fluid (RTF). Additionally, samples from the saliva and tongue were taken. For the tongue, a sterile cotton swab (Nuova Aptaca, Canelli, Italy) was wiped for $10 \mathrm{~s}$ at the back of the tongue. The tip of this cotton swap was transferred to an Eppendorf tube with $1 \mathrm{ml}$ RTF. Approximately $5 \mathrm{ml}$ of unstimulated saliva was collected, from which $100 \mu \mathrm{l}$ was dispersed in $900 \mu \mathrm{l}$ RTF. The presence of Porphyromonas gingivalis, Prevotella intermedia, Fusobacterium nucleatum and Aggregatibacter actinomycetemcomitans in these samples was determined by quantitative PCR assay (qPCR). Bacterial DNA was extracted by using the DNeasy Tissue Kit (QIAGEN Ltd) according to the manufacturers' instruction. A quantitative PCR (qPCR) assay based on the 16s rRNA gene was performed with a CFX96 Real-Time System (Biorad). The Taqman $5^{\prime}$ nuclease assay PCR method was used for detection and quantification of bacterial DNA. Quantification was based on a standard curve.

\section{3 | Treatment protocol}

At the baseline visit, after recording the clinical measurements and taking the microbiological samples, patients were given oral hygiene instructions, and a full-mouth prophylaxis was carried out. Subsequently, a mechanical debridement of the peri-implant sites was performed under local anaesthesia. This was carried out with the Satelec P5 Newtron XS BLED (Acteon) with specific tips (PH1, $\mathrm{PH} 2 \mathrm{~L}$ and $\mathrm{PH} 2 \mathrm{R}$ ), followed by hand instrumentation with titanium curettes. Finally, the peri-implant pockets were subgingivally treated with the Air-N-Go Easy air polisher (Acteon). The treatment session was concluded by a professional topical application of the study drops around the implants with peri-implantitis. In the probiotic group, these were probiotic drops containing L. reuteri DSM 17938 and L. reuteri ATCC PTA 5,289 (10 ${ }^{8}$ CFU of each strain/5 drops) (BioGaia $A B$ ), and in the control group, the placebo drops without bacteria were used. The placebo drops were identical in taste, texture and appearance to the probiotic lozenges. Additionally, probiotic and placebo lozenges were distributed to the patients according to the study group they were assigned to. The patients of the probiotic group received probiotic lozenges containing L. reuteri DSM 17938 and L. reuteri ATCC PTA $5289\left(10^{8}\right.$ CFU of each strain/lozenge) (BioGaia $A B$ ). The patients in the placebo group were handed out lozenges that were identical in appearance, texture and taste, except that live bacteria were excluded. To examine the adherence, the patients were asked to bring back the empty containers in which the study medication was packed at the 12 weeks consultation. Besides this twelve-week follow-up, patients were seen 6 weeks and 24 weeks after the baseline visit. Clinical data were recorded during the baseline, 12- and 24-week visit. Microbiological samples were collected at these time points and additionally at the 6-week follow-up.

\subsection{Statistical methods}

Clinical variables where analysed by means of a linear mixed model with time and treatment as fixed factors and patient as random factor. A normal quantile plot of the residual values and a residual dot plot showed that data were normally and homoscedastically distributed around their expected values.

Bacterial log counts were considered, and counts below quantification limit were considered as censored values (i.e., < quantification limit). Data were analysed by means of a frailty model with time and treatment as fixed factors and patient as random factor. For each of the models, differences between treatments and times were calculated using the fixed effects-estimates of the statistical model and its variance-covariance matrix and $p$-values were corrected for simultaneous hypothesis testing according to Sidak. Missing data at 24 weeks were filled in a forward way.

\section{3 | RESULTS}

The patients participating in this study were recruited between October 2015 and May 2018; the last follow-up consultation took place 24 weeks later, in November 2018. Twenty-three patients were recruited, from which 4 were excluded or lost to follow-up before the 12-week consultation; the results of the remaining 19 patients were used for the analysis. More details about the study course can be found in Figure 1.

The patients consumed on average $1.8 \pm 0.4$ lozenges/day (1.9 \pm 0.3 per day in the test group and $1.6 \pm 0.4$ in the control group). No adverse effects were noted; however, three patients in the control group had minor complaints during the study period reporting a dry mouth or a changed feeling in the oral cavity. Additionally, one patient in the control group and one in the probiotic group indicated that the study medication had a strong (pepper) mint flavour.

\section{1 | Outcomes at the implant level}

The demographics of both study groups can be found in Table 1, the implant characteristics in the online Appendix S1. At baseline the clinical and microbiological characteristics of the selected implants 


\section{Enrollment}

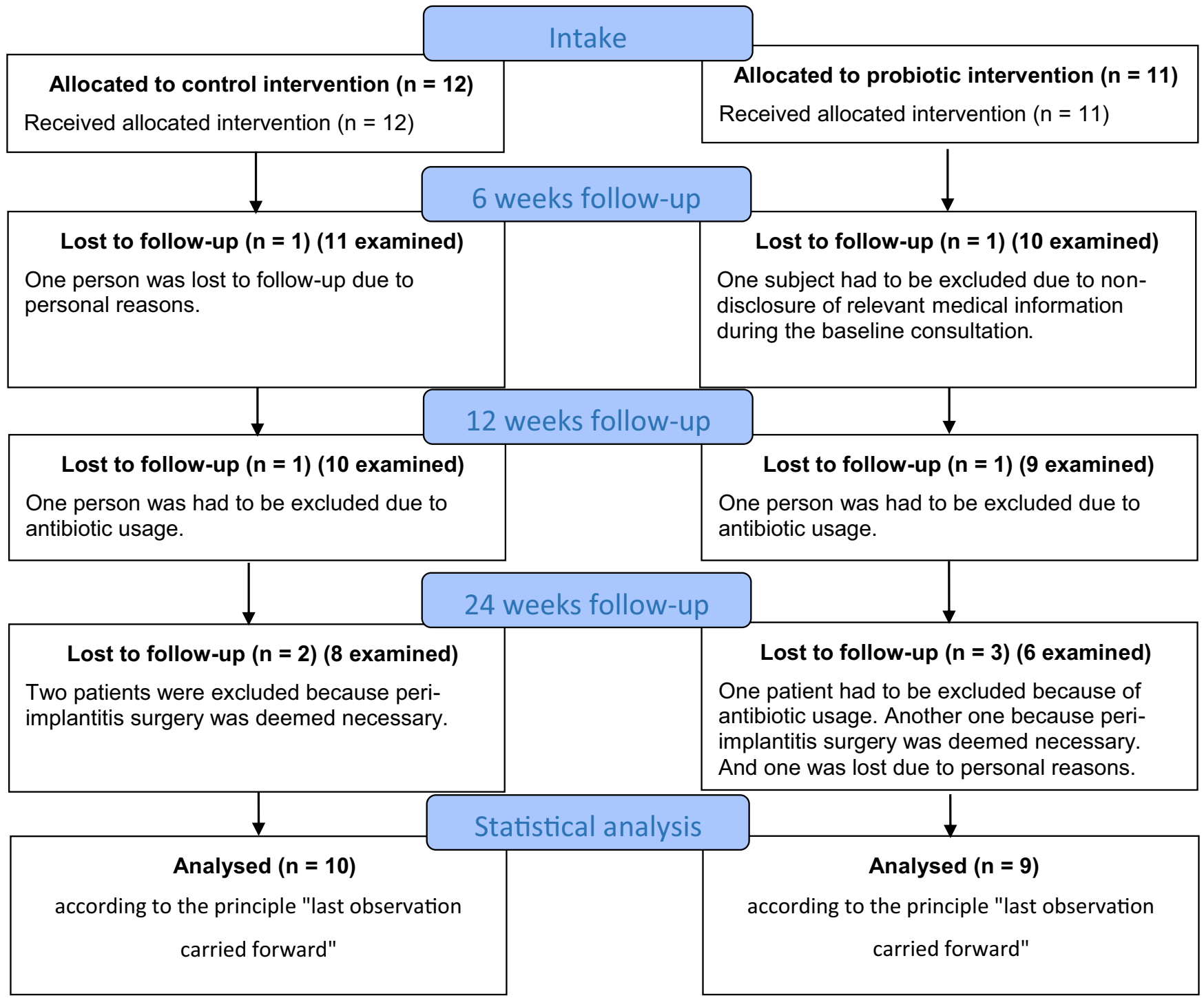

FIGURE 1 Study course

TABLE 1 Demographic characteristics

\begin{tabular}{lll} 
& \multicolumn{2}{l}{ Treatment group } \\
\cline { 2 - 3 } Variable & Probiotic & Control \\
\hline Number of patients & 9 & 10 \\
\hline Number of males & 5 & 4 \\
\hline Number of females & 4 & 6 \\
\hline Number of smokers & 0 & 0 \\
\hline Age (mean \pm SD) (years) & $64 \pm 11$ & $69 \pm 9$ \\
\hline
\end{tabular}

with peri-implantitis were comparable for both the probiotic as the control group. After 12 and 24 weeks, the BoP, mSBI and PPD were significantly improved compared with the baseline measurements, both in the probiotic and the control group. The BoP decreased from $87 \%$ to $59 \%(p<.001)$ for the probiotic group and from $87 \%$ to $53 \%$ $(p<.001)$ for the control group. The modified sulcus bleeding was reduced from $1.92 \pm 0.70$ to $0.89 \pm 0.63(p<.001)$ and from $1.96 \pm 0.79$ to $1.22 \pm 1.07(p<.001)$, respectively. The PPD improved from $5.17 \mathrm{~mm}$ to $4.15 \mathrm{~mm}$ in the probiotic group after 24 weeks $(p<.001)$ and from $5.45 \mathrm{~mm}$ to $4.18 \mathrm{~mm}$ in the control group ( $p<.001)$. More details can be found in Table 2. No statistically significant intergroup differences could be found for these characteristics, neither after 12 weeks nor after 24 weeks. In contrast, the PI only showed statistically significantly reduced values during the follow-up visits in the probiotic, but not in the control group. The decrease in PI was therefore significantly better in the probiotic group compared with the control group ( $p<.001$ at 12 weeks and $p=.002$ at 24 weeks).

Only two implants from the control group achieved the desired outcome and could therefore classified as peri-implant healthy: no more bleeding on probing around the implant. In the test group, no implants met the criteria for being classified as healthy. 
TABLE 2 Clinical characteristics of the implants in the probiotic group versus the implants in the control group displayed as mean or delta $(\Delta)$ (difference with baseline value) and standard deviation (SD)

\begin{tabular}{|c|c|c|c|c|c|c|c|}
\hline Variable & Time point & \multicolumn{4}{|c|}{ Treatment group } & $\begin{array}{l}p \text {-value } \\
\text { For mean }\end{array}$ & For Delta \\
\hline \multicolumn{8}{|l|}{ BOP (\%) } \\
\hline \multirow[t]{2}{*}{ Overall } & Baseline & $87 \pm 23 \%$ & & $87 \pm 22 \%$ & & .999 & \\
\hline & 24 weeks & $59 \pm 32 \%^{a}$ & $-27 \pm 23 \%$ & $53 \pm 39 \%^{a}$ & $-33 \pm 27 \%$ & .998 & .876 \\
\hline \multicolumn{8}{|c|}{ Modified sulcus bleeding index } \\
\hline \multirow[t]{3}{*}{ Overall } & Baseline & $1.92 \pm 0.70$ & & $1.96 \pm 0.79$ & & .999 & \\
\hline & 12 weeks & $1.14 \pm 0.88^{a}$ & $-0.65 \pm 0.86$ & $0.89 \pm 0.86^{a}$ & $-0.92 \pm 0.66$ & .988 & .717 \\
\hline & 24 weeks & $0.89 \pm 0.63^{a}$ & $-0.93 \pm 0.67$ & $1.22 \pm 1.07^{\mathrm{a}}$ & $-0.56 \pm 0.97$ & .972 & .178 \\
\hline Overall & 24 weeks & $4.15 \pm 0.96^{a}$ & $-1.02 \pm 0.69$ & $4.18 \pm 1.26^{\mathrm{a}}$ & $-1.27 \pm 1.00$ & .999 & .801 \\
\hline \multicolumn{8}{|c|}{ Plaque index (\%) } \\
\hline \multirow[t]{3}{*}{ Overall } & Baseline & $15 \pm 13 \%$ & & $8 \pm 21 \%$ & & .924 & \\
\hline & 12 weeks & $3 \pm 7 \%^{\mathrm{a}}$ & $-11 \pm 14 \%$ & $11 \pm 19 \%$ & $+3 \pm 23 \%$ & .833 & $<.001$ \\
\hline & 24 weeks & $2 \pm 6 \%^{a}$ & $-13 \pm 14 \%$ & $7 \pm 14 \%$ & $-2 \pm 16 \%$ & .980 & .002 \\
\hline
\end{tabular}

Note: Bold: significant intergroup difference.

${ }^{a}$ Significant intragroup difference compared to the baseline value.

No major intra- nor intergroup differences were recorded regarding the microbiological counts of four know periodontal pathogens. This is shown in Table 3.

\subsection{Outcomes at the full-mouth level}

No baseline differences could be found regarding the overall plaque and bleeding scores of the included patients (Table 4). Both statistically significantly decreased after 12 and 24 weeks in both the probiotic and control group. No intergroup differences concerning FMBS could be detected, but the decrease of the FMPS was significantly better in the probiotic group compared with the control group. Twelve weeks after the study start, the FMPS was $10 \%$ reduced in the probiotic group compared with $5 \%$ in the control group ( $p=.001$ ). At 24 week this difference was even more pronounced with $14 \%$ decrease of FMPS in the probiotic group and only $4 \%$ in the control group $(p<.001)$.

\section{DISCUSSION}

The objective of this study was to examine the added effect of probiotics on the non-surgical treatment of peri-implantitis. In order to include a group of patients that was as homogeneous as possible, strict inclusion criteria were proposed. On one hand, we tried to exclude possible non-plaque-related factors affecting peri-implantitis: at least $2 \mathrm{~mm}$ keratinized mucosa should be present around the implant and no restorative problems should be diagnosed around the implant. On the other hand, we targeted a group of patients with initial peri-implantitis, since we hypothesized that it would be easier in these patients to control the peri-implant inflammation. For each patient, only one implant was included (even if more implants were affected) to avoid the influence of host-related factors.

Significantly better clinical variables after non-surgical peri-implantitis treatment were shown in this trial. However, the added value of probiotics in this therapy could not be shown. The only statistically significant difference that was demonstrated was a higher decrease in $\mathrm{PI}$ at the level of the peri-implantitis sites in the patients in the probiotic group compared with the control group. When looking at this parameter at the whole mouth level (FMPS), no differences in plaque score could be seen between both groups. However, statistically significant better scores for FMBS could be noted. No microbiological differences could be found, neither intranor intergroup.

This study thus failed to reproduce the additional healing effect seen by the use of probiotics in non-surgical peri-implantitis treatment by previous clinical trials (Galofre et al., 2018; Tada et al., 2018). However, both studies have a very specific design, which makes it not possible to compare the results of this study with those trials. Galofré and co-workers examined a very specific population 
WI LEY- CIINICAL ORAL IMPLANTS REEARCH

TAB LE 3 Microbiological (log-transferred) outcome measures: mean and standard deviation values at baseline and the differences ( $\Delta$ ) after 6, 12 and 24 weeks

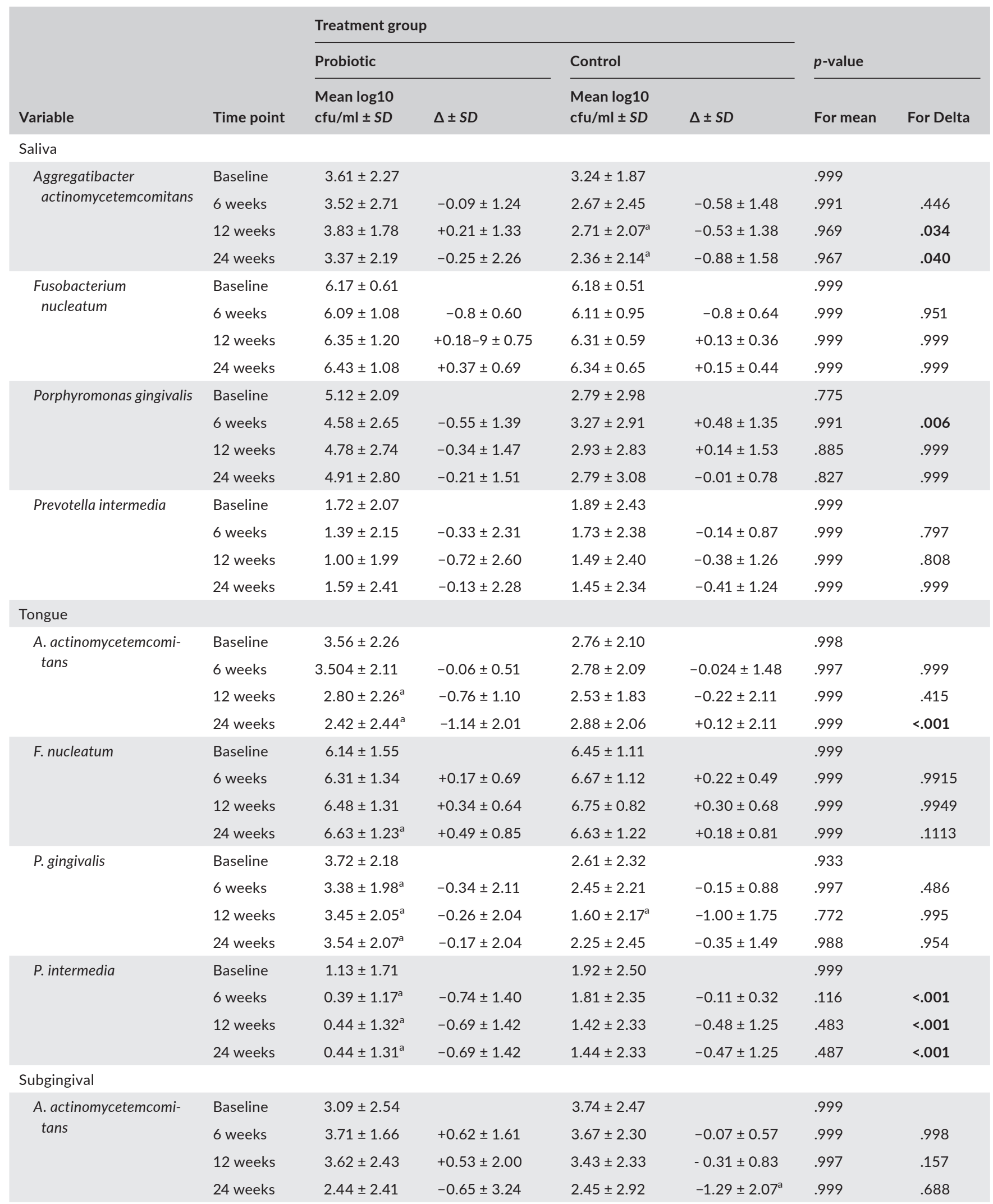


TABLE 3 (Continued)

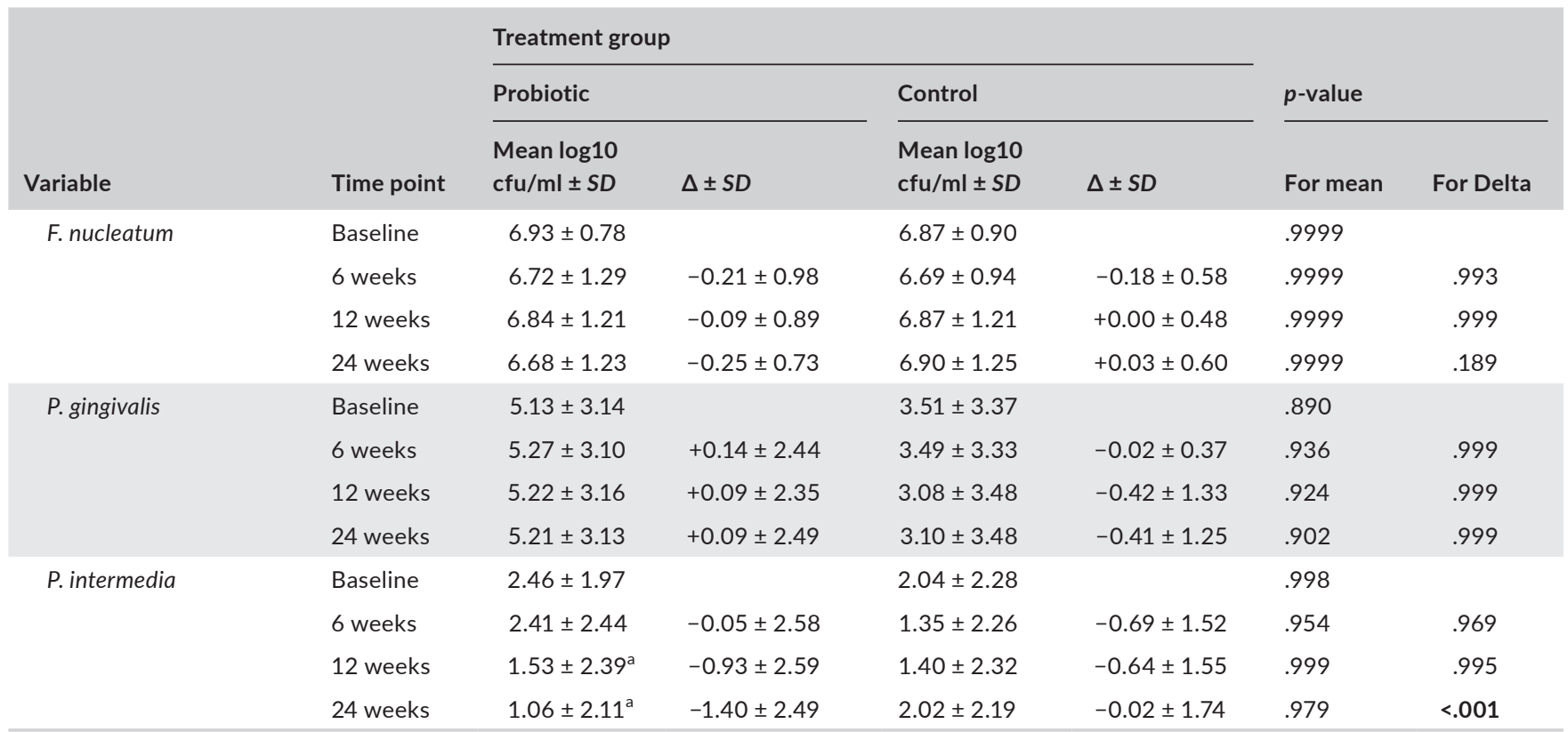

Note: Bold: significant intergroup difference.

${ }^{a}$ Significant intragroup difference compared to the baseline value.

only including former periodontitis patients and Tada et al. used antibiotics as pre-treatment.

The lack of adverse effects reported confirmed again the safety of this dual-strain probiotic. The only side-effect reported was an altered sensation of the oral cavity by three patients; however, since all three of them were included in the control group, it can be concluded that this is not related to the active study component. It is assumed that this is rather due to the increased attention for the oral cavity due to participation in a clinical trial.

This study showed that the clinical characteristics of peri-implantitis sites are statistically significantly improved by debridement and oral hygiene instructions. It is however important to examine the clinical implication of this statistical improvement. In this patient population, it was seen that these improvements only led to a completely healthy peri-implant tissue in 2 out of 19 patients. It thus seems difficult to completely resolve the peri-implant inflammation with only non-surgical debridement. This is in line with earlier results showing that it is difficult to control peri-implant inflammation without a surgical phase, even when peri-implant mucositis was diagnosed (Lang, Salvi, \& Sculean, 2019; Peña et al., 2019). Moreover, a recent retrospective analysis showed that also in the long-term non-surgical procedures are insufficient to

TABLE 4 Clinical characteristics of the group assigned to the probiotic products versus the group assigned to the control products displayed as mean or delta $(\Delta)$ (difference with baseline value) and standard deviation (SD)

\begin{tabular}{|c|c|c|c|c|c|c|c|}
\hline \multirow{2}{*}{ Variable } & \multirow{2}{*}{ Time point } & \multicolumn{4}{|c|}{ Treatment group } & & \\
\hline & & \multicolumn{2}{|l|}{ Probiotic } & \multicolumn{2}{|l|}{ Control } & \multicolumn{2}{|l|}{$p$-Value } \\
\hline \multicolumn{8}{|l|}{ FMBS (\%) } \\
\hline Overall & Baseline & $30 \pm 10 \%$ & & $21 \pm 13 \%$ & & .5640 & \\
\hline \multicolumn{8}{|l|}{ FMPS (\%) } \\
\hline \multirow[t]{3}{*}{ Overall } & Baseline & $29 \pm 11 \%$ & & $30 \pm 14 \%$ & & .9999 & \\
\hline & 6 weeks & $25 \pm 11 \%$ & $-4 \pm 13 \%$ & $24 \pm 8 \%^{a}$ & $-6 \pm 11 \%$ & .9999 & .999 \\
\hline & 12 weeks & $20 \pm 11 \%^{a}$ & $-8 \pm 10 \%$ & $21 \pm 10 \%^{a}$ & $-8 \pm 12 \%$ & .9999 & .999 \\
\hline
\end{tabular}

Note: Bold: significant intergroup difference.

${ }^{a}$ Significant intragroup difference compared to the baseline value. 
prevent further bone loss at peri-implantitis sites (Karlsson et al., 2019). Keeping these results in mind, there are two lines of thought about the non-surgical treatment of peri-implant inflammatory diseases. Or, we have not yet found the most optimal treatment for removing the biofilm during non-surgical treatment. Or, non-surgical treatment is simply not enough to reverse the peri-implant inflammation.

Future research should take both lines of thinking into account. Concerning the first remark, the use of the plastic tips $(\mathrm{PH} 1, \mathrm{PH} 2 \mathrm{~L}$ and $\mathrm{PH} 2 \mathrm{R}$ ) could be criticized since these are rather thick and may not be the best-suited instruments to reach the bottom of the pocket. For future research, it would be preferable to use titanium tips for the ultrasonic debridement (such as IP1, IP2L, IP2R, IP3L and IP3R from Acteon). Additionally, the repeated application of the probiotic drops could also be considered to improve the contact time locally, that is between the inflamed region and the study product. This could be done at home by the patients with a syringe and blunt needle.

Seeing the non-surgical treatment of peri-implantitis only as a preliminary treatment of peri-implantitis also has consequences for future research. In light of this, the use of probiotics to keep the peri-implant situation stable after the surgical phase could be examined. If we suppose that a surgical phase is almost always needed and we add to this the non-linear and accelerating loss of tissue in peri-implantitis sites (Schwarz et al., 2018), there seems no purpose to target "initial" peri-implantitis. At the study start, these inclusion criteria were chosen in analogy with previous studies (Bassetti et al., 2014; Schar et al., 2013); however, at this moment it is clear that initial peri-implantitis is neither a specific histological, nor a separate clinical entity.

Possible drawbacks of this study are the fact that different implant brands and types were included, dietary probiotics were not explicitly prohibited and the small sample size. Although a large diversity of implants is a clinical reality, this may have influenced the study outcomes. Specific implant characteristics (brand, roughness, chemical coating, thread pitch, etc.) can, after all, influence the occurrence and rate of peri-implantitis (Derks et al., 2016). Additionally, since at the study start, no randomized controlled trials were available examining probiotics in the non-surgical treatment of peri-implantitis, we chose to perform a pilot study that can be used in the future for sample size calculations. A post hoc power calculation showed that 180 and even more than 20,000 patients are needed to obtain a statistically significant difference between both groups for $\mathrm{mSBI}$ and PPD respectively.

Finally, future research should not only focus on clinical and microbiological factors, but also on inflammatory markers such as IL-1 $\beta$, IL- 6 and IL-8. Increased insight in the underlying mechanisms of healing will provide a better understanding of these processes and help to improve our therapies. Certainly, when probiotics are investigated as a therapy, inflammatory markers should be monitored, since modulation of the inflammatory response is more and more suggested as a possible action mechanism of probiotics.

\section{ACKNOWLEDGEMENTS}

The study products (the probiotic as well as the placebo products) used in this clinical trial were kindly provided by BioGaia $A B$. The Air-N-Go Easy air polisher was provided by Acteon. We hereby thank our secretary, Andrea Van Obberghen for blinding the study medication.

\section{CONFLICT OF INTEREST}

Wim Teughels received fees for lecturing on probiotics from BioGaia AB.

\section{AUTHOR CONTRIBUTIONS}

IL, WT and MQ conceived the ideas. IL collected the data. MP conducted the microbiological analysis. IL and WT analysed the data (in collaboration with a statistician). IL wrote the manuscript. IL, WT, MP and MQ revised and approved the manuscript in its current state.

\section{ORCID}

Isabelle Laleman (iD https://orcid.org/0000-0001-9662-8238

\section{REFERENCES}

Bassetti, M., Schar, D., Wicki, B., Eick, S., Ramseier, C. A., Arweiler, N. B. ... Salvi, G. E. (2014). Anti-infective therapy of peri-implantitis with adjunctive local drug delivery or photodynamic therapy: 12-month outcomes of a randomized controlled clinical trial. Clinical Oral Implants Research, 25(3), 279-287. https://doi.org/10.1111/clr.12155

Berglundh, T., Armitage, G., Araujo, M. G., Avila-Ortiz, G., Blanco, J. Camargo, P. M., ... Zitzmann, N. (2018). Peri-implant diseases and conditions: Consensus report of workgroup 4 of the 2017 world workshop on the classification of periodontal and peri-implant diseases and conditions. Journal of Periodontology, 89(Suppl. 1), S313-s318. https://doi.org/10.1002/jper.17-0739

Della Riccia, D. N., Bizzini, F., Perilli, M. G., Polimeni, A., Trinchieri, V., Amicosante, G., \& Cifone, M. G. (2007). Anti-inflammatory effects of Lactobacillus brevis (CD2) on periodontal disease. Oral Diseases, 13(4), 376-385. https://doi.org/10.1111/j.1601-0825.2006.01291.x

Derks, J., Schaller, D., Hakansson, J., Wennstrom, J. L., Tomasi, C., \& Berglundh, T. (2016). Effectiveness of implant therapy analyzed in a Swedish population: Prevalence of peri-implantitis. Journal of Dental Research, 95(1), 43-49. https://doi.org/10.1177/0022034515 608832

Flichy-Fernandez, A. J., Ata-Ali, J., Alegre-Domingo, T., Candel-Marti, E., Ata-Ali, F., Palacio, J. R., \& Penarrocha-Diago, M. (2015). The effect of orally administered probiotic Lactobacillus reuteri-containing tablets in peri-implant mucositis: A double-blind randomized controlled trial. Journal of Dental Research, 50(6), 775-785. https://doi. org/10.1111/jre.12264

Galofre, M., Palao, D., Vicario, M., Nart, J., \& Violant, D. (2018). Clinical and microbiological evaluation of the effect of Lactobacillus reuteri in the treatment of mucositis and peri-implantitis: A triple-blind randomized clinical trial. Journal of Dental Research, 53(3), 378-390. https://doi.org/10.1111/jre.12523

Hallstrom, H., Lindgren, S., Widen, C., Renvert, S., \& Twetman, S. (2016). Probiotic supplements and debridement of peri-implant mucositis: A randomized controlled trial. Acta Odontologica Scandinavica, 74(1), 60-66. https://doi.org/10.3109/00016357.2015.1040065

Hallstrom, H., Lindgren, S., Yucel-Lindberg, T., Dahlen, G., Renvert, S., \& Twetman, S. (2013). Effect of probiotic lozenges on 
inflammatory reactions and oral biofilm during experimental gingivitis. Acta Odontologica Scandinavia, 71(3-4), 828-833. https://doi. org/10.3109/00016357.2012.734406

Harini, P. M., \& Anegundi, R. T. (2010). Efficacy of a probiotic and chlorhexidine mouth rinses: A short-term clinical study. Journal of Indian Society of Pedodontics and Preventive Dentistry, 28(3), 179-182. https ://doi.org/10.4103/0970-4388.73799

Heitz-Mayfield, L. J. A., \& Salvi, G. E. (2018). Peri-implant mucositis. Journal of Clinical Periodontology, 45, S237-S245. https://doi. org/10.1111/jcpe.12953

Hill, C., Guarner, F., Reid, G., Gibson, G. R., Merenstein, D. J., Pot, B., ... Sanders, M. E. (2014). Expert consensus document. The international scientific association for probiotics and prebiotics consensus statement on the scope and appropriate use of the term probiotic. Nature Reviews Gastroenterology \& Hepatology, 11(8), 506-514. https://doi. org/10.1038/nrgastro.2014.66

Ince, G., Gursoy, H., Ipci, S. D., Cakar, G., Emekli-Alturfan, E., \& Yilmaz, S. (2015). Clinical and biochemical evaluation of lozenges containing lactobacillus Reuteri as an adjunct to non-surgical periodontal therapy in chronic periodontitis. Journal of Periodontology, 86(6), 746-754. https://doi.org/10.1902/jop.2015.140612

Iniesta, M., Herrera, D., Montero, E., Zurbriggen, M., Matos, A. R., Marin, M. J., ... Sanz, M. (2012). Probiotic effects of orally administered Lactobacillus reuteri -containing tablets on the subgingival and salivary microbiota in patients with gingivitis. A randomized clinical trial. Journal of Clinical Periodontology, 39(8), 736-744.

Karlsson, K., Derks, J., Håkansson, J., Wennström, J. L., Petzold, M., \& Berglundh, T. (2019). Interventions for peri-implantitis and their effects on further bone loss: A retrospective analysis of a registry-based cohort. Journal of Clinical Periodontology, 46(8), 872-879. https://doi.org/10.1111/jcpe.13129

Krasse, P., Carlsson, B., Dahl, C., Paulsson, A., Nilsson, A., \& Sinkiewicz, G. (2005). Decreased gum bleeding and reduced gingivitis by the probiotic Lactobacillus reuteri. Swedish Dental Journal, 30, 55-60.

Lang, N. P., Salvi, G. E., \& Sculean, A. (2019). Nonsurgical therapy for teeth and implants-When and why? Periodontology 2000, 79(1), 1521. https://doi.org/10.1111/prd.12240

Mombelli, A., van Oosten, M. A., Schurch, E. Jr, \& Land, N. P. (1987). The microbiota associated with successful or failing osseointegrated titanium implants. Oral Microbiology and Immunology, 2(4), 145-151. https://doi.org/10.1111/j.1399-302X.1987.tb00298.x

Mongardini, C., Pilloni, A., Farina, R., Di Tanna, G., \& Zeza, B. (2017). Adjunctive efficacy of probiotics in the treatment of experimental peri-implant mucositis with mechanical and photodynamic therapy: A randomized, cross-over clinical trial. Journal of Clinical Periodontology, 44(4), 410-417. https://doi.org/10.1111/jcpe.12689

Morales, A., Carvajal, P., Silva, N., Hernandez, M., Godoy, C., Rodriguez, G., ... Gamonal, J. (2016). Clinical effects of Lactobacillus rhamnosus in non-surgical treatment of chronic periodontitis: A randomized placebo-controlled trial with 1-year follow-up. Journal of Periodontology, 87(8), 944-952. https://doi.org/10.1902/jop.2016.150665

Peña, M., Barallat, L., Vilarrasa, J., Vicario, M., Violant, D., \& Nart, J. J. C. O. I. (2019). Evaluation of the effect of probiotics in the treatment of peri-implant mucositis: A triple-blind randomized clinical trial. Clinical Oral Investigations, 23(4), 1673-1683. https://doi.org/10.1007/ s00784-018-2578-8

Sajedinejad, N., Paknejad, M., Houshmand, B., Sharafi, H., Jelodar, R., Shahbani Zahiri, H., \& Noghabi, K. A. (2018). Lactobacillus salivarius NKO2: A potent probiotic for clinical application in mouthwash.
Probiotics and Antimicrobial Proteins, 10(3), 485-495. https://doi. org/10.1007/s12602-017-9296-4

Schar, D., Ramseier, C. A., Eick, S., Arweiler, N. B., Sculean, A., \& Salvi, G. E. (2013). Anti-infective therapy of peri-implantitis with adjunctive local drug delivery or photodynamic therapy: Six-month outcomes of a prospective randomized clinical trial. Clinical Oral Implants Research, 24(1), 104-110. https://doi.org/10.1111/j.1600-0501.2012.02494.x

Schlagenhauf, U., Jakob, L., Eigenthaler, M., Segerer, S., Jockel-Schneider, Y., \& Rehn, M. (2016). Regular consumption of Lactobacillus reuteri-containing lozenges reduces pregnancy gingivitis: An RCT. Journal of Clinical Periodontology, 43(11), 948-954. https://doi. org/10.1111/jcpe.12606

Schwarz, F., Derks, J., Monje, A., \& Wang, H.-L. (2018). Peri-implantitis. Journal of Clinical Periodontology, 45, S246-S266. https://doi. org/10.1111/jcpe.12954

Shimauchi, H., Mayanagi, G., Nakaya, S., Minamibuchi, M., Ito, Y., Yamaki, K., \& Hirata, H. (2008). Improvement of periodontal condition by probiotics with Lactobacillus salivarius WB21: A randomized, double-blind, placebo-controlled study. Journal of Clinical Periodontology, 35(10), 897-905.

Tada, H., Masaki, C., Tsuka, S., Mukaibo, T., Kondo, Y., \& Hosokawa, R. (2018). The effects of Lactobacillus reuteri probiotics combined with azithromycin on peri-implantitis: A randomized placebo-controlled study. Journal of Prosthodontic Research, 62(1), 89-96. https://doi. org/10.1016/j.jpor.2017.06.006

Tekce, M., Ince, G., Gursoy, H., Dirikan Ipci, S., Cakar, G., Kadir, T., \& Yilmaz, S. (2015). Clinical and microbiological effects of probiotic lozenges in the treatment of chronic periodontitis: A 1-year follow-up study. Journal of Clinical Periodontology, 42(4), 363-372. https://doi. org/10.1111/jcpe.12387

Teughels, W., Durukan, A., Ozcelik, O., Pauwels, M., Quirynen, M., \& Haytac, M. C. (2013). Clinical and microbiological effects of Lactobacillus reuteri probiotics in the treatment of chronic periodontitis: A randomized placebo-controlled study. Journal of Clinical Periodontology, 40(11), 1025-1035.

Vicario, M., Santos, A., Violant, D., Nart, J., \& Giner, L. (2013). Clinical changes in periodontal subjects with the probiotic Lactobacillus reuteri Prodentis: A preliminary randomized clinical trial. Acta Odontologica Scandinavica, 71(3-4), 813-819. https://doi. org/10.3109/00016357.2012.734404

Vivekananda, M. R., Vandana, K. L., \& Bhat, K. G. (2010). Effect of the probiotic Lactobacilli reuteri (Prodentis) in the management of periodontal disease: A preliminary randomized clinical trial. Journal of Oral Microbiology, 2.

\section{SUPPORTING INFORMATION}

Additional supporting information may be found online in the Supporting Information section.

How to cite this article: Laleman I, Pauwels M, Quirynen M, Teughels $\mathrm{W}$. The usage of a lactobacilli probiotic in the non-surgical therapy of peri-implantitis: A randomized pilot study. Clin Oral Impl Res. 2019;00:1-9. https://doi. org/10.1111/clr.13555 